\title{
Gastroparesis in Non-Diabetics: Associated Conditions and Possible Risk Factors
}

\author{
Yousef Nassar ${ }^{\mathrm{a}, \mathrm{c}}$, Seth Richter ${ }^{\mathrm{b}}$
}

\begin{abstract}
Background: Gastroparesis is a syndrome characterized by delayed gastric emptying in the absence of any mechanical cause. While often associated with diabetes mellitus, most cases of gastroparesis are idiopathic. The purpose of the present paper is to review the co-morbid conditions that most likely associate with non-diabetic gastroparesis.

Methods: The Healthcare Cost and Utilization Project: Nationwide Inpatient Sample (HCUP-NIS) data were used from the year 2013 2014 and the Apriori algorithm was run on this subset of patients to identify what co-morbid conditions are most likely associated with gastroparesis.
\end{abstract}

Results: Notable conditions that were found to be most closely linked with gastroparesis were: chronic pancreatitis, end stage renal disease, irritable bowel syndrome, systemic lupus erythematosus, fibromyalgia, and venous thromboembolism. Some of the implications of these findings are briefly discussed.

Conclusions: Gastroparesis is strongly associated with multiple medical conditions which may be potential targets for treatment.

Keywords: Idiopathic gastroparesis; Non-diabetic gastroparesis

\section{Introduction}

Gastroparesis is a syndrome characterized by delayed gastric emptying in the absence of any mechanical cause. It is estimated that gastroparesis affects up to $4 \%$ of the US population and inpatient care for patients with severe gastroparesis costs $\$ 7,000$ per month [1]. In other words, gastroparesis is a relatively common disorder and comes with high costs.

Gastroparesis is commonly associated with neuropathy secondary to diabetes mellitus. Although there is limited data

Manuscript submitted June 19, 2018, accepted July 18, 2018

aDepartment of Medicine, Albany Medical Center, Albany, NY, USA bivision of Gastroenterology, Albany Medical Center, Albany, NY, USA 'Corresponding Author: Yousef Nassar, 43 New Scotland Avenue, MC-17, A1bany, NY 12208, USA. Email: nassary@amc.edu

doi: https://doi.org/10.14740/gr1060w investigating conditions associated with gastroparesis in nondiabetic patients, most cases are idiopathic (35\%). Other etiologies include surgery (13\%), viral infections (8.2\%), Parkinson's disease $(7.5 \%)$, miscellaneous $(6.2 \%)$, collagen vascular disease (4.8\%), and intestinal pseudo-obstruction (4.1\%) [1].

The most common major presenting symptoms for gastroparesis include nausea, vomiting, and early satiety but the pattern of symptoms is often determined by the underlying cause of gastroparesis. For example, while nausea is a predominant symptom in all patients with gastroparesis, vomiting is typically more prevalent and severe in patients with diabetic gastroparesis in comparison to other causes [2-4]. Less common symptoms such as postprandial fullness, upper abdominal pain, or abdominal distention may present as well. To complicate matters, there is little clinical and pathophysiologic data on patients with gastroparesis, which often leads to chronic morbidity and frequent hospitalizations.

The pathogenesis of idiopathic gastroparesis has not been studied as extensively as that of diabetic gastroparesis, despite the fact that idiopathic gastroparesis is very common. At the present time, some pathophysiological differences between idiopathic and diabetic gastroparesis have been reported. For instance, individuals with idiopathic gastroparesis show a lower degree of vagal neuropathy and less vagal damage compared to diabetic gastroparesis patients [1]. The two types of gastroparesis also present different histomorphological characteristics but the significance of these differences is not clear [5]. The inflammatory or immune-mediated processes associated with idiopathic gastroparesis can alter important elements that allow the normal gastric motor function, such as changes of the nerves, muscle cells, and interstitial cells of Cajal [6].

In terms of clinical presentation, idiopathic and diabetic gastroparesis are similar, though several differences exist. $\mathrm{Pa}-$ tients with diabetic gastroparesis are 13 years older and heavier at the onset of symptoms and hospitalized more frequently compared to patients with idiopathic gastroparesis. In patients with diabetic gastroparesis, vomiting is often the main symptom that prompts evaluation while in patients with idiopathic gastroparesis, abdominal pain is more common. Gastric retention is greater in patients with type 1 diabetes and gastroparesis than in patients with idiopathic gastroparesis whereas severe early satiety and excessive fullness are more common in patients with idiopathic gastroparesis [7]. Idiopathic gastroparesis tends to be more associated with pain [5].

It is worth mentioning that $62 \%$ of individuals with idiopathic gastroparesis reveal a history of physical and sexual abuse; on the other hand, studies have yet to establish whether 
Table 1. Demographic Information for Non-Diabetic Patients With Gastroparesis in Our Patient Population (Mean Age = 51.2)

\begin{tabular}{lll}
\hline Demographic information & Total $(\%)$ & Odds ratio $\mathbf{9 5 \%}$ CI) \\
\hline Female & $19,700(71.5)$ & $1.471(1.429-1.515) *$ (compared to male controls) \\
Male & $7,843(28.5)$ & \\
Caucasian & $19,558(74.8)$ & $0.923(0.889-0.959) *$ (compared to Caucasian controls) \\
African Americans & $3,806(14.6)$ & $0.670(0.638-0.705) *$ (compared to Caucasian controls) \\
Hispanic & $1,858(7.1)$ & $0.458(0.406-0.517) *($ compared to Caucasian controls) \\
Asian or Pacific Islander & $274(1.0)$ & $0.788(0.656-0.946) *($ compared to Caucasian controls) \\
Native American & $120(0.5)$ & $0.668(0.611-0.73) *$ (compared to Caucasian controls) \\
\hline
\end{tabular}

${ }^{*} \mathrm{P}<0.001$.

an association exists between the history of abuse and delayed gastric emptying [7].

Menopausal status and the phase of the menstrual cycle have been associated with gastric emptying. For instance, the luteal phase (days 18 - 20) of the menstrual cycle is associated with a slower gastric emptying compared to the follicular phase (days 8 - 10). During the luteal phase, estrogen and progesterone are at high levels and these two hormones are thought to have an inhibitory effect on gastric motility. It has also been shown that postmenopausal women engaged in sex hormone replacement therapy have slower gastric emptying compared to men, an observation that supports the role of estrogen and progesterone as inhibitors of gastric emptying. On the other hand, there have also been reports of delayed gastric emptying during the follicular phase of the menstrual cycle, which questions the importance of hormones in explaining gender differences in gastroparesis prevalence.

There have also been reports on gender differences in proximal stomach affecting motility and perception, as relaxation of the proximal stomach that occurs postprandially is prolonged in women compared to men. A relevant clinical finding is that symptoms of early satiety and nausea are more common in females than in males, at almost $2: 1$ ratio. These findings suggest that it is important to compare females with symptoms of gastric dysfunctions using gastric emptying parameters derived in normal women and not in normal men [8].

In practice, gastroparesis is a clinical diagnosis, but the definitive diagnostic test is a gastric emptying study such as scintigraphy $[2,3,9]$. The treatment options for gastroparesis vary from conservative management, such as dietary modifications and medication, to more invasive surgical options. Dietary modifications focus on eating smaller semi-solid meals and avoiding foods that exacerbate symptoms. Medications for persistent symptoms include antiemetics and prokinetic agents, such as metoclopramide, to improve gastric emptying. In patients with refractory gastroparesis, surgical options include intrapyloric botulinum toxin injections, placement of a feeding jejunostomy tube, or implantation of a gastric electrical stimulator $[3,10,11]$.

Developing more effective gastroparesis management strategies could not only reduce the costs associated with gastroparesis but also improve aspects of patients' quality of life (QOL). For instance, a recent research found that patients with gastroparesis have significantly lower scores of QOL as assessed by a validated QOL measure (SF-36) and the McGill Pain Questionnaire compared to patients with serious medical conditions and scores similar to patients that have both serious physical and mental health conditions [12].

Differentiating between the etiologies of gastroparesis may allow developing strategies that could increase QOL in gastroparesis patients. For instance, it has been suggested that treatment for gastroparesis may produce different responses depending on whether gastroparesis occurs in diabetic or nondiabetic patients. However, according to the findings of a retrospective study, response to treatment (antiemetics, prokinetics, tricyclic antidepressants, analgesic and invasive interventions) does not correlate with the type of gastroparesis (non-diabetic or diabetic) or the presence of delayed emptying but correlates with stomach distention, bloating, and the Gastroparesis Cardinal Symptom Index (GCSI). This study puts into question the relevance of etiology in the management of gastroparesis, at least in diabetic vs. non-diabetic conditions [13]. However, it is worth noticing that treatment for the two conditions are not usually directed at correcting the underlying etiology [14] for which exploring the causes of idiopathic gastroparesis may still offer valuable insights that could be used to guide treatments that target the cause of the symptoms.

\section{Materials and Methods}

For this retrospective study, the Healthcare Cost and Utilization Project: Nationwide Inpatient Sample (HCUP-NIS) data were used from the year 2013 - 2014. The database was searched for all non-diabetic patients diagnosed with gastroparesis (ICD 9 diagnostic code 536.6). The Apriori algorithm was then run on this subset of patients to identify what co-morbid conditions are most likely associated with gastroparesis. The Apriori algorithm is used for mining the frequency of data sets and is designed to find associations in databases. It works by identifying frequent single item sets in a database and then stepwise expanding each of those sets, keeping the new larger sets if the terms appear in sufficient frequency in the database. In this study, we employed the Apriori algorithm as a "bottom-up" approach: each subset is expanded individually and the subset of gastroparesis patients is tested against each new iteration of the 
Table 2. Diagnoses Associated With Gastroparesis in Non-Diabetics Compared To Controls

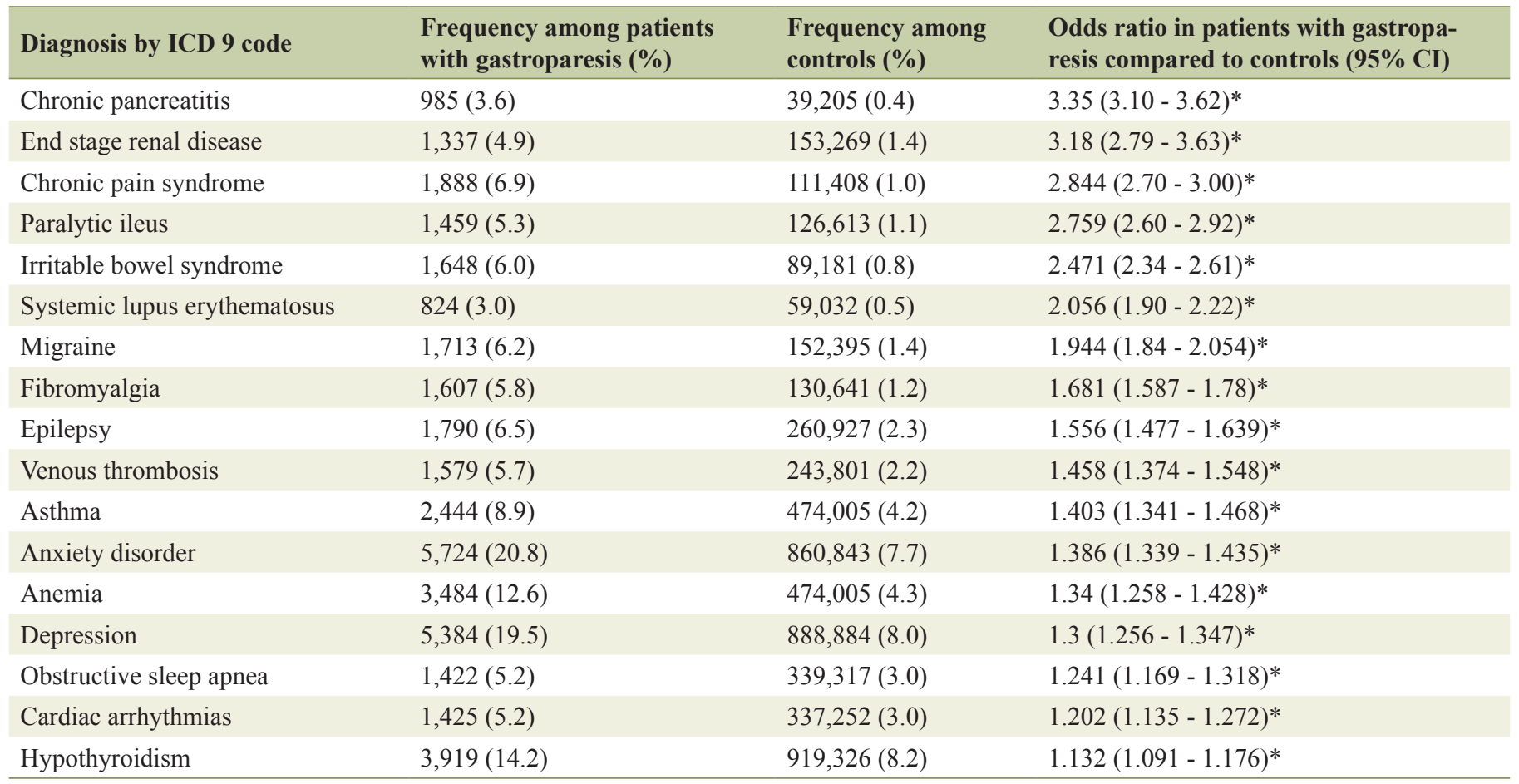

${ }^{*} \mathrm{P}<0.001$.

data. The algorithm terminates when no more associations can be found $[15,16]$. The diagnoses that were found when we ran the Apriori algorithm on the HCUP-NIS data were compared to controls using multivariate logistic regression and the primary disease associations to gastroparesis were determined. These results were further studied to determine what the distribution of race, age and gender was for the patient population studied.

\section{Results}

A total of 27,543 patients with the diagnosis of idiopathic gastroparesis were included in this study. In this patient set, it was found that 19,700 were female and 7,843 were male. Women were more likely to be diagnosed with gastroparesis when compared to male controls with an odds ratio (OR) of $1.47(\mathrm{P}<0.001)$, which is consistent with the current statistics regarding gastroparesis $[1,2]$. Table 1 demonstrates the racial and ethnic distribution of the patient set with gastroparesis. As seen, gastroparesis occurs most frequently in Caucasian patients, followed at a significant distance by African Americans and Hispanic patients. Gastroparesis occurs significantly less frequently in African Americans with an OR of 0.92 (P $<0.001)$ compared to Caucasian patients. Table 2 conversely lists the medical diseases with the highest association with gastroparesis in this patient set. Psychiatric conditions such as depression and anxiety were most frequently associated with gastroparesis. Notable conditions that were found to be most closely linked with gastroparesis were: chronic pancreatitis with OR $3.35(\mathrm{P}<0.001)$, end stage renal disease with OR
$3.18(\mathrm{P}<0.001)$, chronic pain syndrome with $\mathrm{OR} 2.844(\mathrm{P}$ $<0.001$ ), and irritable bowel syndrome (IBS) with an OR of $2.47(\mathrm{P}<0.001)$. Other diagnoses that were also frequently associated with gastroparesis were systemic lupus erythematosus (SLE), fibromyalgia, hypothyroidism, chronic kidney disease, obstructive sleep apnea, cardiac arrhythmias as well as a history of venous thromboembolism.

\section{Discussion}

Typically seen in patients with poorly controlled diabetes, gastroparesis is a dysmotility disorder of the stomach that causes a delay in the process of digestion, specifically food emptying into the intestine from the stomach. It presents with nonspecific symptoms, such as nausea and vomiting with or without abdominal distention $[10,17,18]$. In recent literature, it has been found more frequently in non-diabetics; however, it is difficult to identify reasons for this primarily due to a lack of understanding of the causes or the conditions that are associated with gastroparesis. In this study, we identified several conditions associated with gastroparesis in a set of non-diabetics, including anxiety, depression, chronic pancreatitis, hypothyroidism, end stage renal disease, SLE, fibromyalgia, IBS, and electrolyte disturbances. The pathophysiology behind gastroparesis is not completely understood, however, further study of its association with the above medical conditions may offer new insights $[3,9,17]$.

One of the conditions with a high association with gastroparesis, psychiatric illness, is also a common comorbidity 
seen in patients with other intestinal-related illnesses, such as IBS and gastroesophageal reflux. These patients are often prescribed antidepressants for anxiety and depression. Up to $70 \%$ of patients with gastroesophageal reflux have some level of comorbid anxiety. In addition, IBS patients also present with increased anxiety, most commonly in refractory cases. Studies have shown a higher frequency of nausea, vomiting, bloating and post-prandial fullness in patients with depression and anxiety. Patients with moderate to severe depression who are treated for gastroparesis are nearly $50 \%$ less likely to show improvement $[18,19]$. Conversely, in gastroparesis patients who show an improvement with prokinetic medications, there was a decreased frequency of depression and anxiety [20-22]. As many as $18 \%$ and $31 \%$ of gastroparesis patients suffer from severe depression and severe anxiety, respectively, leaving a sizeable population with a limited prognosis [23-25]. Despite this strong evidence linking gastroparesis to depression and anxiety, there is currently not enough evidence that can help ascertain which interventions would be most helpful or how they would improve outcomes. Further studies are needed to determine if proper management of these associated psychiatric conditions would also improve response to treatment of gastroparesis.

Because gastrointestinal motility is also in part affected by thyroid hormone levels, it is important to study the association between hypothyroidism and gastroparesis. Patients with hypothyroidism often present with symptoms of hypomotility, such as flatulence and constipation, as well as nausea and vomiting. Several tools which measure gastric emptying time (GET) are used to diagnose gastrointestinal hypomotility. Specifically, a barium swallow test is the diagnostic radiographic test of choice $[9,10]$. In patients with gastroparesis and chronic hypothyroidism, pathologic examination reveals that the gastrointestinal mucosa is pale, edematous and thick with a mononuclear cell infiltration. In contrast, patients with a limited course of hypothyroidism typically have normal mucosa. Despite the above evidence of this correlation, more studies are needed in order to determine the particular mechanism behind this association.

Another condition linked with gastroparesis is SLE. SLE can involve any part of the gastrointestinal tract (GI) or hepatobiliary system. Some of the manifestations of GI symptoms in SLE include oral lesions, mesenteric vasculitis, gastritis, pancreatitis, inflammatory bowel disease, enteropathy, and esophageal dysmotility. Signs of small bowel involvement occur in greater than 50 percent of patients and often manifest chronically as pseudo-obstruction, bacterial overgrowth, and malnutrition. In fact, patients will often show signs of obstruction without a mechanical cause of obstruction. Additionally, they will present with bloating, flatulence, diarrhea, and weight loss due to malabsorption. Studies attempting to alleviate gastroparesis by treating the underlying SLE have shown limited success, suggesting that either the gastroparesis is irreversible or shows a delayed response to treatment in SLE patients [17]. Symptomatic treatment is the most common approach, such as antibiotics, bowel rest, nutritional support, decompression, and prokinetic agents [26]. Currently, gastroparesis is seen as a possible late-onset or severe manifestation of SLE, however more studies are needed to provide further evidence.

Gastroparesis is associated with severe reflux, dyspepsia, or, both. It is estimated that about $25 \%$ of patients with gastroesophageal reflux disease (GERD) have gastroparesis [27]. The reported prevalence of slower rates of gastric emptying in GERD patients differs according to the methodology used by different studies. There has been an improvement in this direction in the last years, as international control values for scintigraphic gastric emptying have been adopted [28], which should allow for better estimations of prevalence in the future.

Delayed gastric emptying has been reported in a few patients with IBS; however, there are important aspects to be considered in this regard. For instance, according to one research study, IBS patients without dyspepsia had normal gastric emptying of solids and gastroparesis symptoms were common only in IBS patients with dyspepsia. The same study found a $66 \%$ prevalence of functional dyspepsia in IBS patients. The observed association between dyspeptic symptoms and delayed gastric emptying is not a direct one, as there was no detected abnormality of gastric emptying of solids in many functional dyspepsia patients [29].

Other estimates suggest that between $23 \%$ and $87 \%$ of patients with IBS have functional dyspepsia and between $13 \%$ and $87 \%$ of patients with dyspepsia have IBS. The important differences in the estimated ranges are likely explained by the differences in the diagnostic criteria used and the small size of the samples [30]. These findings suggest that gastroparesis symptoms are common in IBS patients but in most cases, they appear in patients with IBS and dyspepsia.

Chronic pain is thought to have a major contribution to lowering the QOL in patients with gastroparesis. It has been hypothesized that affect plays an important role in influencing symptom severity. Gastroparesis often affects QOL by lowering individuals' ability or willingness to engage in social relationships with family, friends, and/or colleagues and reducing the quality of professional life. People with gastroparesis are more likely to be unemployed and have incomes below the national average, something which has also been observed in other disorders associated with chronic pain [31].

Management of pain in gastroparesis is challenging, as dietary and prokinetic therapies do not provide clear benefits. While tricyclic antidepressants (TCAs), selective serotonin reuptake inhibitors (SSRIs), serotonin/norepinephrine reuptake inhibitors, gabapentin, and pregabalin can reduce acutely pain thresholds in healthy individuals, it is not clear whether they have similar effects in the clinical population. Opioids, on the other hand, have a negative impact on gastric emptying and an addictive potential [31].

Fibromyalgia is more likely to affect women. Data suggest $50 \%$ of patients with fibromyalgia experience symptoms of functional dyspepsia. An examination of the gastric motility in patients recently diagnosed with fibromyalgia found no significant difference between patients and control group in terms of gastric emptying rates but did found a longer lag phase in the fibromyalgia patients. The longer lag phase results in a late decompression of the stomach, which explains the commonality of gastrointestinal complains in fibromyalgia patients. Gastroparesis is thought to reflect an abnormal function of the nervous vague and it is possible that the same is true for patients with fibromyalgia [32].

Gastric motor function in patients with chronic renal failure 
(CRF) remains unclear, as it has not been determined whether gastric emptying is delayed in these patients or not. According to a study, patients with CRF show gastric lower gastric motility, including impaired gastric myoelectrical activity and delayed gastric emptying [33]. While gastric hypomotility may be an important underlying factor in gastrointestinal symptoms experienced by CRF patients, the mechanisms through which CRF influences gastric motility is not understood. It is possible that in CRF certain gastrointestinal hormones that modulate motility such as cholecystokinin and gastrin are increased. More so, cytokines and uremic toxins that affect the autonomic and enteric nervous system may change the electrical properties of gastric smooth muscle or pacemaker cells [33].

Severe gastrointestinal motility disorders are associated with intravenous access clots or deep venous thrombosis. One report on a sample of 62 patients with gastroparesis found that $89 \%$ of the patients had detectable hypercoagulable risk factors while $40 \%$ of the patients had a history of abnormal clotting. This finding suggests patients with severe gastroparesis should receive a coagulation evaluation [34].

Because chronic pancreatitis includes symptoms that are similar to gastroparesis, diagnostic confusion sometimes occurs, especially in the case of small-duct chronic pancreatitis which is more difficult to diagnose. More so, the presence of gastroparesis in patients with chronic pancreatitis may negatively impact the effectiveness of pancreatic enzyme therapy by failing to deliver proteases into the duodenum [35]. As the etiology of gastroparesis is still unclear, further studies are required in order to determine what significance these associated conditions have. This study shows that gastroparesis has a strong correlation with mental illness, systemic diseases, such as SLE, and other gastrointestinal manifestations, such as gastroesophageal reflux and IBS, but further research is needed to fully understand how the pathophysiology of gastroparesis contributes to this.

There were multiple limitations and strengths to this study. The limitations include the fact that this study was a retrospective cohort study. The nature of the data provides limited information on study patients but was able to provide data required for our study. Using a large national database provided a large study sample size which provided adequate data for this study. The method of performing this study provided diagnoses associated with idiopathic gastroparesis but did not provide information regarding treatment or effect of management of associated conditions on symptoms of gastroparesis. Further studies may provide more information on the effect of management of associated conditions with the improvement of gastroparesis symptoms.

\section{Conflict of Interest}

None.

\section{Financial Support}

No source of funding was provided for this study.

\section{References}

1. Hasler WL. Gastroparesis - current concepts and considerations. Medscape J Med. 2008;10(1):16.

2. Daruwala C, Mercogliano G, Harder TP. Gastrointestinal manifestations of systemic lupus erythematosus and scleroderma. Clinical Medicine. Gastroenterology. 2009;2:CGast-S2264.

3. Hasler WL, Parkman HP, Wilson LA, Pasricha PJ, Koch KL, Abell TL, Snape WJ, et al. Psychological dysfunction is associated with symptom severity but not disease etiology or degree of gastric retention in patients with gastroparesis. Am J Gastroenterol. 2010;105(11):2357-2367.

4. Ortiz-Torrent N. Psychiatric aspects of gastroparesis. Gastroparesis. 2012:303-313. Humana Press.

5. Camilleri M, Grover M, Farrugia G. What are the important subsets of gastroparesis? Neurogastroenterol Motil. 2012;24(7):597-603.

6. Bielefeldt K. Gastroparesis: concepts, controversies, and challenges. Scientifica (Cairo). 2012;2012:424802.

7. Masaoka T, Tack J. Gastroparesis: current concepts and management. Gut Liver. 2009;3(3):166-173.

8. Parkman HP. Idiopathic gastroparesis: gender aspects. Principles of Gender-Specific Medicine (Second Edition). 2010. p. 326-330.

9. Jian R, Ducrot F, Piedeloup C, Mary JY, Najean Y, Bernier JJ. Measurement of gastric emptying in dyspeptic patients: effect of a new gastrokinetic agent (cisapride). Gut. 1985;26(4):352-358.

10. Janssens J, Peeters TL, Vantrappen G, Tack J, Urbain JL, De Roo M, Muls E, et al. Improvement of gastric emptying in diabetic gastroparesis by erythromycin. Preliminary studies. N Engl J Med. 1990;322(15):1028-1031.

11. Nguyen L. Idiopathic Gastroparesis. Gastroparesis. 2012. p. 205-211. Humana Press.

12. Yunus TM, Mathis C, Grabbe KM, Heinberg LJ, Lacy BE. Quality of life in patients with gastroparesis. The American Journal of Gastroenterology. 2003;98(s9):S57.

13. Anaparthy R, Pehlivanov N, Grady J, Yimei H, Pasricha PJ. Gastroparesis and gastroparesis-like syndrome: response to therapy and its predictors. Dig Dis Sci. 2009;54(5):1003-1010.

14. O'Donovan D, Feinle-Bisset C, Jones K, Horowitz M. Idiopathic and Diabetic Gastroparesis. Curr Treat Options Gastroenterol. 2003;6(4):299-309.

15. Agrawal R, Srikant R. Fast algorithms for mining association rules. In Proc. 20th int. conf. very large data bases, VLDB. 1994;1215:487-499.

16. Pasricha PJ, Yates KP, Nguyen L, Clarke J, Abell TL, Farrugia G, Hasler WL, et al. Outcomes and Factors Associated With Reduced Symptoms in Patients With Gastroparesis. Gastroenterology. 2015;149(7):1762-1774 e11764.

17. Enweluzo C, Aziz F. Gastroparesis: a review of current and emerging treatment options. Clin Exp Gastroenterol. 2013;6:161-165.

18. Haans JJ, Masclee AA. Review article: The diagnosis and management of gastroparesis. Aliment Pharmacol Ther. 2007;26(Suppl 2):37-46. 
19. Parkman HP, Yates K, Hasler WL, Nguyen L, Pasricha PJ, Snape WJ, Farrugia G, et al. Clinical features of idiopathic gastroparesis vary with sex, body mass, symptom onset, delay in gastric emptying, and gastroparesis severity. Gastroenterology. 2011;140(1):101-115.

20. Cherian D, Paladugu S, Pathikonda M, Parkman HP. Fatigue: a prevalent symptom in gastroparesis. Dig Dis Sci. 2012;57(8):2088-2095.

21. Smith DS, Williams CS, Ferris CD. Diagnosis and treatment of chronic gastroparesis and chronic intestinal pseudo-obstruction. Gastroenterol Clin North Am. 2003;32(2):619-658.

22. Woodhouse S, Hebbard G, Knowles SR. Psychological controversies in gastroparesis: A systematic review. World J Gastroenterol. 2017;23(7):1298-1309.

23. Chou J, Zheng H, Bertken R. Gastroparesis in systemic lupus erythematosus: a case report. Journal of Medical Cases. 2013;4:314-315.

24. Parkman HP, Hasler WL, Fisher RS, American Gastroenterological A. American Gastroenterological Association technical review on the diagnosis and treatment of gastroparesis. Gastroenterology. 2004;127(5):1592-1622.

25. Reardon DP, Yoo PS. Levothyroxine Tablet Malabsorption Associated with Gastroparesis Corrected with Gelatin Capsule Formulation. Case Rep Endocrinol. 2016;2016:1316724.

26. Hasler WL, Wilson LA, Parkman HP, Koch KL, Abell TL, Nguyen L, Pasricha PJ, et al. Factors related to abdominal pain in gastroparesis: contrast to patients with predominant nausea and vomiting. Neurogastroenterol Motil. 2013;25(5):427-438, e300-421.

27. Noar MD, Noar E. Gastroparesis associated with gastroesophageal reflux disease and corresponding reflux symptoms may be corrected by radiofrequency ablation of the cardia and esophagogastric junction. Surg Endosc. 2008;22(11):2440-2444.

28. Buckles DC, Sarosiek I, McMillin C, McCallum RW. Delayed gastric emptying in gastroesophageal reflux disease: reassessment with new methods and symptomatic correlations. Am J Med Sci. 2004;327(1):1-4.

29. Stanghellini V, Tosetti C, Barbara G, De Giorgio R, Cogliandro L, Cogliandro R, Corinaldesi R. Dyspeptic symptoms and gastric emptying in the irritable bowel syndrome. Am J Gastroenterol. 2002;97(11):2738-2743.

30. Frissora CL, Koch KL. Symptom overlap and comorbidity of irritable bowel syndrome with other conditions. Curr Gastroenterol Rep. 2005;7(4):264-271.

31. Bielefeldt K, Raza N, Zickmund SL. Different faces of gastroparesis. World J Gastroenterol. 2009;15(48):60526060.

32. Erdogan S, Gurer G, Afsin H, Kucukzeybek Y. Evaluation of gastric emptying rate in patients with fibromyalgia: a case control study. Mod Rheumatol. 2011;21(2):174-177.

33. Hirako M, Kamiya T, Misu N, Kobayashi Y, Adachi H, Shikano M, Matsuhisa E, et al. Impaired gastric motility and its relationship to gastrointestinal symptoms in patients with chronic renal failure. J Gastroenterol. 2005;40(12):1116-1122.

34. Lobrano A, Blanchard K, Rock W, Johnson W, Schmieg B, Borman K, Araghizadeh F, et al. Assessing thrombosis risk in patients with idiopathic, diabetic, and postsurgical gastroparesis. Adv Ther. 2006;23(5):750-768.

35. Chowdhury RS, Forsmark CE, Davis RH, Toskes PP, Verne GN. Prevalence of gastroparesis in patients with small duct chronic pancreatitis. Pancreas. 2003;26(3):235238. 